\section{Metastatic tumor antigen 3 is a direct corepressor of the Wnt4 pathway}

\author{
Hao Zhang, , ,3 Rajesh R. Singh, ${ }^{1,3}$ \\ Amjad H. Talukder, ${ }^{1,3}$ and Rakesh Kumar ${ }^{1,2,4}$ \\ ${ }^{1}$ Molecular and Cellular Oncology, The University of Texas \\ M.D. Anderson Cancer Center, Houston, Texas 77030, USA; \\ ${ }^{2}$ Molecular and Cellular Biology, Baylor College of Medicine, \\ Houston, Texas 77030, USA
}

Here we show that expression of MTA3 inhibits ductal branching in virgin and pregnant murine transgenic mammary glands. MTA3 also suppresses the Wnt4 pathway and, thus, these findings parallel phenotypic changes in Wnt4-null mice. MTA3 represses Wnt4 transcription and Wnt4 secretion, inhibiting Wnt-target genes in mammary epithelial cells. Accordingly, knockdown of endogenous MTA3 stimulates Wnt4 expression and Wnt cellular targets. The MTA3-NuRD (nucleosome remodeling and deacetylase) complex physically interacts with the Wnt4 chromatin in an HDAC-dependent manner, leading to suppression of the Wnt4 gene and Wnt4-dependent morphogenesis. These findings identify MTA3 as an upstream physiologic repressor of Wnt4 in mammary epithelial cells.

Supplemental material is available at http://www.genesdev.org.

Received June 21, 2006; revised version accepted September 6, 2006.

Multiprotein complexes, including the vertebrate $\mathrm{Mi}-2 /$ nucleosome remodeling and deacetylase (Mi-2/NuRD) complex, have been implicated in chromatin remodeling in normal and pathologic states (Kumar 2003; Kumar et al. 2003). The NuRD complex is composed of subunits specializing in ATP-dependent chromatin remodeling and histone deacetylation, and involved in chromatin compaction and transcriptional repression. In spite of the similarity in the mode of action among the subunits, the functional specialization of each is bestowed by the presence of different metastatic tumor antigen (MTA) family members in the NuRD complex. MTAl was the first member of the family, initially identified as a differentially expressed gene in breast cancer cell lines (Pencil et al. 1993). Subsequently, the two other members, MTA2 and MTA3, were identified and found to be present in distinct NuRD complexes, which lack functional redundancy. Using cancer cell lines, MTA3 was shown to be an estrogen-responsive gene whose expression depends on the presence of estrogen receptor $\alpha(\mathrm{ER} \alpha)$ (Fujita et al. 2003; Mishra et al. 2004).

[Keywords: MTA3; WNT4; mammary gland, lactation]

${ }^{3}$ These authors contributed equally to this work.

${ }^{4}$ Corresponding author.

E-MAIL rkumar@mdanderson.org; FAX (713) 745-3792

Article published online ahead of print. Article and publication date are online at http://www.genesdev.org/cgi/doi/10.1101/gad.1461706.
Our understanding of MTA3 functions is primarily derived from the cancer cell line-based models. For example, MTA3 interacts with the promoter of the transcription repressor Snail and down-regulates its expression in a HDAC-dependent manner in cancer cell lines. Since Snail is known to promote epithelial-to-mesenchymal transitions primarily via down-regulating the expression of E-cadherin, MTA3-induced repression of Snail has been linked to the differentiation and increased expression of E-cadherin in human breast cancer cell lines (Fujita et al. 2003). The second known target of MTA3 was BCL6. MTA3 Mi2/NuRD complex deacetylates BCL6, and regulates differentiation of B lymphocytic cell lines (Fujita et al. 2004). Although cell linebased findings have provided important insights into the biological activity of MTA3, its physiologic role in whole mammary gland development remains unknown.

The Wnt family of secreted proteins, which bind to specific cell surface receptors, influences mammary gland development and tumorigenesis. Wnt1 and Wnt3 were the first Wnt family members implicated in mouse mammary carcinomas (Nusse and Varmus 1992). Wnt4 is maximally expressed in the mammary epithelium during early to mid pregnancy (Bradbury et al. 1995). Mutant Wnt4-null mammary glands in early pregnancy showed a specific defect in progesterone-induced ductal side branching, implicating Wnt4 as a downstream effector of progesterone signaling (Brisken et al. 2000). In an attempt to achieve a greater clarity of MTA3's biological functions in a physiological setting, here we investigated the effect of targeted overexpression of MTA3 in mouse mammary gland. We found that Wnt4 is a direct target of MTA3 and that phenotypic changes in the MTA3 transgenic mammary glands remarkably resemble those from the Wnt4-null mice.

\section{Results and Discussion}

MTA3 deregulation in mouse mammary epithelium leads to a defective ductal branching

To understand the influence of MTA3 on mammary gland development and morphogenesis, we generated a transgenic mice expressing human T7-MTA3 cDNA under the control of the mouse mammary tumor virus long terminal repeat (Supplementary Fig. S1A). Three transgenic founder mouse lines with variable copy number of MTA3 transgene were generated (Supplementary Fig. $\mathrm{S} 1 \mathrm{~B}, \mathrm{C})$. Transgene T7-MTA3 was expressed in a variety of organs with the highest expression in the mammary gland (Supplementary Fig. S1D). Immunofluorescence of a mammary gland with an anti-T7 Ab further revealed a mosaic nature of MTA3 transgene expression in the nucleus of epithelial cells (Supplementary Fig. S1E). Expression of T7-MTA3 in mammary glands was further confirmed by immunoprecipitation followed by Western blot analysis using an anti-T7 mAb (Supplementary Fig. S1F).

To determine the contribution of MTA3 to mammary gland development, we analyzed the status of ductal branching in the virgin glands from wild-type and MTA3-TG female mice. Glands were isolated and whole mounted, and compared at the developmental stage $(4$ wk) and mature stage (10-18 wk). At 4 wk of age, termi- 


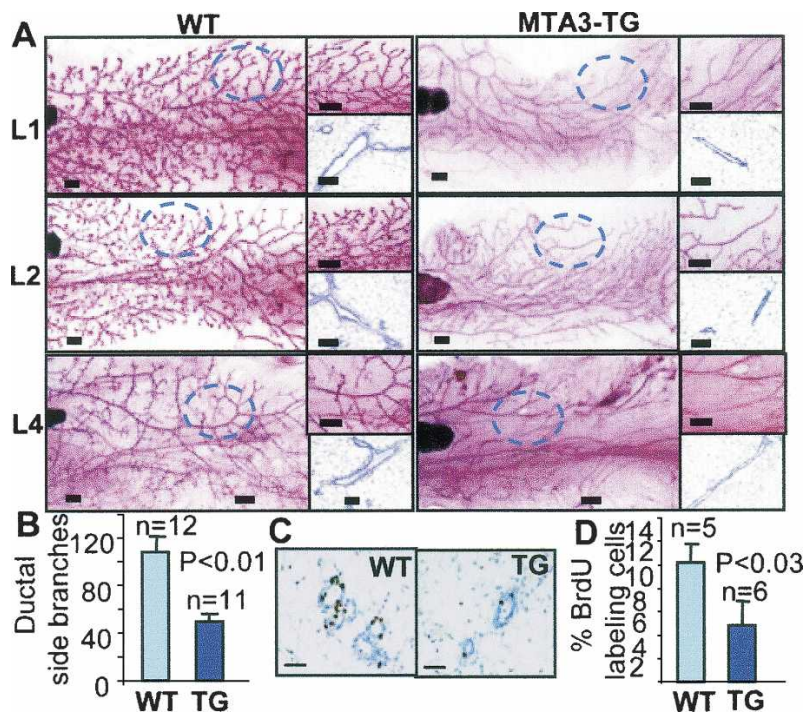

Figure 1. MTA3 leads to a defective ductal branching in mouse mammary epithelium. (A) Whole-mount analysis of virgin $16-\mathrm{wk}$ mammary glands from the wild-type (WT) and the MTA3-TG mice. Bars: whole-mount, $500 \mu \mathrm{m}$; H\&E, $50 \mu \mathrm{m}$. (B) Quantitation of ducta side branches. (C) BrdU labeling of epithelium in virigin 16-wk mice. Bars, $50 \mu \mathrm{m}$. (D) Quantification of BrdU-labeling index of epithelium.

nal end buds were present and appeared normal in the transgenic mice (data not shown). At maturity, the ducts had reached nearly to the end of the fat pads; however, significantly less secondary and tertiary ductal branches were observed in the MTA3-TG mouse glands. These phenotypic changes were observed in mammary glands in three founder lines (Fig. 1A). The number of ductal side branches in wild-type branches $(107.8 \pm 13.7, n=12)$ was higher than the number in transgenic branches $(48.8 \pm 7.1, n=11)$ (Fig. 1B).

\section{Ductal branch defect is due to impaired epithelial cell proliferation}

To determine whether the defect in mammary gland development was the result of reduced cell proliferation or increased cell death, BrdU incorporation and TdT-mediated dUTP-X nick-end labeling (TUNEL) assays were performed. Wild-type and MTA3 virgin females (16-wkold) were administered BrdU for its incorporation into DNA (Fig. 1C). The proliferation rate of the MTA3-TG mammary epithelium $(4.8 \% \pm 2.7, n=6)$ was approximately half that of the wild-type mammary epithelium $(10.2 \% \pm 2.2, n=5)$ (Fig. 1D). There were no significant differences in the apoptosis rates, measured by TUNEL assay, between wild-type and MTA3 mammary glands (data not shown). These results suggested that MTA3 status might be a determinant of mammary epithelium proliferation. Interestingly, there was no effect of MTA3TG on the levels of Snail (a direct target of MTA3 in cancer cell lines) or E-cadherin in the mammary gland (Supplementary Fig. S2A,B). The noticed lack of any significant effect of MTA3-TG on the level of Snail expression is not in line with a previous report showing Snail repression in cell lines (Fujita et al. 2003). These differences may be possibly due to the lack of a normal epithelial cell line in the previous study or MTA3 represses Snail expression only in cancer cell line or both.
Defective Wnt4 pathway in mammary gland epithelial cells of MTA3-TG mice

Since progesterone receptors (PRs) and Wnt signaling are the major regulators of ductal branching in mammary glands (Robinson et al. 2000) and because PR and Wnt knockout females exhibit a defect in mammary gland development (Lydon et al. 1995) similar to that in the MTA3-TG females, we next determined whether deregulation of MTA3 affects PR or Wnt expression and function in mammary epithelium. We first examined PR expression in the wild-type and MTA3-TG virgin mammary glands. IHC analysis revealed that wild-type cells were $34.7 \% \pm 12.7$ positive for PR versus $31.2 \% \pm 14.2$ in the MTA3-TG cells. Similarly, levels of ER $\alpha$-positive cells $(47.2 \% \pm 9.2$ wild-type cells and $46.3 \% \pm 8.3 \mathrm{TG}$ cells were ER positive), showed no variation between the wild-type and TG virgin mammary glands, suggesting that the observed phenotypic changes in the MTA3-TG mice are not due to the change in the ER or PR status (Supplementary Fig. S3A,B).

Next we examined the levels of $\beta$-catenin, a key component of the canonical Wnt pathway. We found that $\beta$-catenin levels were much lower in the MTA3-TG mammary glands than in the wild-type glands (Fig. 2A), suggesting a potential role of MTA3 in repressing Wnt pathway. Accordingly, the levels of cyclin D1, one of the Wnt targets, were reduced to $14.6 \% \pm 8.6, n=5$ ) in the MTA3-TG as compared with $35.4 \% \pm 7.3$ cyclin D1positive cells in the wild-type mammary epithelial cells (Fig. 2B). Interestingly, we also discovered a considerable decrease in the in situ levels of Wnt4 as well as of WNT4 protein in the MTA3-TG mammary gland when compared with the wild-type mammary gland (Fig. 2C,D).

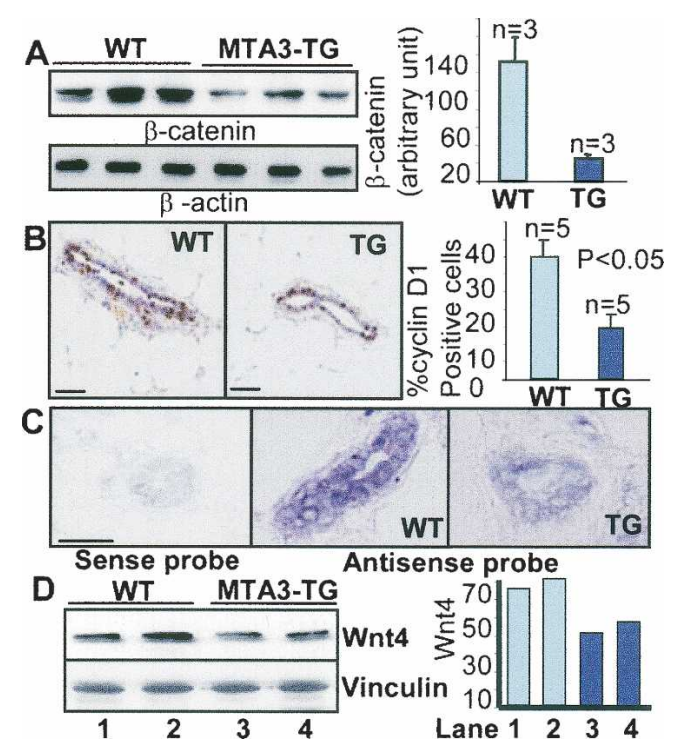

Figure 2. Impaired Wnt pathway in the MTA3-TG mammary tissue. (A) Western blot analysis of $\beta$-catenin in the virgin mammary tissue. (B) IHC analysis of cyclin D1 in the wild-type (WT) and TG mammary tissues. Bars, $100 \mu \mathrm{m} .(C)$ In situ hybridization of digoxigenin-labeled Wnt 4 cDNA antisense transcripts to mammary tissue sections. Bars, $100 \mu \mathrm{m}$. (D) Western blot analysis of Wnt4 from tissue lysates. Mammary glands used were from the virgin 12-wkold female mice. 


\section{Secretion of Wnt4 ligand is suppressed by MTA3}

To elucidate the role of MTA3 in the Wnt pathway in mammary gland epithelium, we next generated stable pooled clones of T7-MTA3 in the HC11 murine mammary epithelial cell line (Fig. 3A), which has been extensively used as a valid model to study the proliferative and differentiating effects of Wnt genes (Humphreys and Rosen 1997; Civenni et al. 2003). To assess the effect of MTA3-TG on the WNT4 pathway, we compared the levels of Wnt4 mRNA in the HC11/MTA3 cells with the levels in the control pcDNA clones, and found a drastic reduction in the level of WNT4 mRNA in the HC11/ MTA3 cells (Fig. 3B). The observed decreased WNT4 expression in the HC11/MTA3 cells was accompanied by reduced Wnt4 secretion in the conditioned media as compared with the control pcDNA cells (Fig. 3C). These findings suggested a negative regulatory role of MTA3 on Wnt4 expression and secretion in mammary epithelial cells.

\section{MTA3 suppresses the canonical Wnt pathway}

The drastic down-regulation of Wnt4 secretion in HC11/ MTA3 cells is expected to inhibit the canonical Wnt pathway. Accordingly, we found a significant reduction in the level of $\beta$-catenin in the HC11/MTA3 clones as compared with the levels in the HC11/pcDNA clones (Fig. 3D). We also found lower levels of phosphorylated Ser9-GSK3 $\beta$ in the HC11/MTA3 clones than in the HC11/pcDNA clones (Fig. 3D) showing more active GSK3 $\beta$, which leads to greater phosphorylation and degradation of $\beta$-catenin in HC11/MTA3 clones. To validate the repression of the canonical Wnt pathway by MTA3, we show that MTA3 suppresses the $\beta$-catenin/LEF signaling using a TOP-Flash-luc activity (Fig. 3E). We also found a significant inhibition in the levels of WISP2
mRNA, a Wnt target gene, in the HC11/MTA3 clones (Fig. 3F).

\section{MTA3 is a physiologic negative regulator of Wnt4 expression}

To further strengthen the noticed causative correlation between the levels of MTA3 and repression of the Wnt4 pathway, we next silenced the endogenous MTA3 expression in the HC11/pcDNA cells by an MTA3-specific small interfering RNA (siRNA) and assessed the status of Wnt4 pathway. We found that a conditional reduction in the amount of MTA3 leads to an enhancement in the levels of Wnt4 and its target WISP2 mRNA (Fig. 3G,H). We next explored the potential involvement of the MTA3/NuRD complex in the repression of Wnt4 expression by MTA3. Treatment of the HC11/MTA3 cells with a general HDAC inhibitor TSA resulted in an increased Wnt4 expression (Fig. 3I) as well as accumulation of the secreted Wnt4 (Fig. 3J) in the HC11/MTA3 cells as compared with its levels in the control cells. These findings suggested that MTA3 represses Wnt4 expression in an HDAC-dependent manner in mammary epithelial cells, and that MTA3 status may be an upstream determinant of the Wnt4 expression, and consequently, functions in mammary epithelial cells.

\section{MTA3/NuRD complex associates with two putative regions of the Wnt4 regulatory chromatin}

The negative regulatory chromatin region of the mouse Wnt4 gene has been mapped up to $7 \mathrm{~kb}$ upstream of the start site (Devagan et al. 2005). To examine the physical interaction of the MTA3/NuRD complex with the Wnt4 chromatin, we carried out a promoter walk for the $10-\mathrm{kb}$ region upstream of the Wnt4 gene using chromatin immunoprecipitation (ChIP) analysis. Initially, ChIP ly-

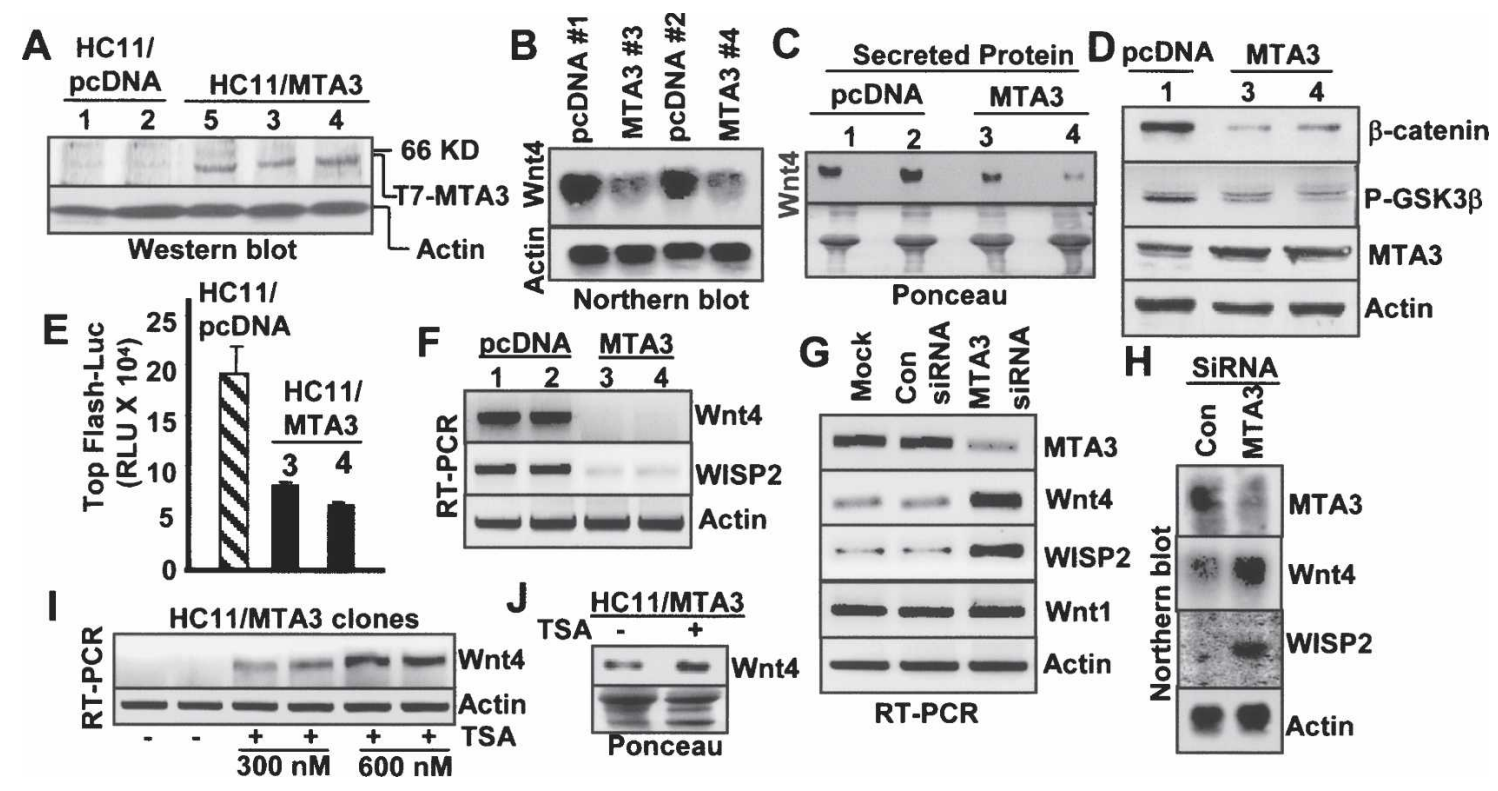

Figure 3. MTA 3 status affects Wnt 4 expression and signaling. $(A)$ Expression of T7-MTA3 protein in the indicated pooled HC11 clones. $(B)$ Northern blot analysis of the Wnt 4 mRNA. $(C)$ Western blot showing Wnt4 protein in the culture media. $(D)$ Western blot analysis of $\beta$-catenin and Ser9 phosho-GSK3 $\beta$. (E) Top-flash-Luc activity in the HC11 clones. (RLU) Relative light unit. (F) RT-PCR analysis of the WISP2 mRNA. $(G, H)$ Effect of MTA3-siRNA on the levels of Wnt4 and WISP2 mRNAs. (I) Effect of TSA on the levels of Wnt4 mRNA by RT-PCR. (J) Effect of TSA on secreted Wnt4 protein. 
sates from the HC11/MTA3 cells were precipitated with an anti-T7 mAb to pull down T7-MTA3 or with IgG as a control and eluted DNA was tested by PCR analysis with primers designed against every 500-base-pair (bp) region of the 10-kb Wnt4 chromatin. This analysis identified two Wnt4 promoter MTA3-interacting regions, encompassing roughly a $1-\mathrm{kb}$ region $(-2681 \mathrm{bp}$ to $-3793 \mathrm{bp}$; i.e., region 6 and 7$)$ and a $1.5-\mathrm{kb}$ region $(-7688 \mathrm{bp}$ to -9352 bp; i.e., regions 15, 16, and 17) (Fig. 4A,B). Since HDACs are integral components of the MTA3/NuRD complex and MTA3 represses WNT4 expression in an HDAC-dependent manner (Fig. 3I,J), we next tested whether HDAC2 is also recruited to the MTA3-interacting Wnt4 chromatin. ChIP analysis with an HDAC2specific antibody showed that HDAC2 was present on the same $1.0-\mathrm{kb}(-2681 \mathrm{bp}$ to $-3793 \mathrm{bp})$ and $1.5-\mathrm{kb}(-7688$ bp to $-9352 \mathrm{bp}$ ) regions of the Wnt4 chromatin as MTA3, raising the possibility of recruitment of the MTA3/ NuRD complex to the above regions of the Wnt4 chromatin (Fig. 4B). ChIP in HC11/MTA3 cells for Mi2, the largest subunit of the NuRD complex, showed the recruitment of $\mathrm{Mi} 2$ to both the $1-\mathrm{kb}$ and the $1.5-\mathrm{kb}$ regions of the Wnt4 chromatin (Fig. 4B, lower panel). In the $1-\mathrm{kb}$ region, a greater association of $\mathrm{Mi} 2$ was observed with the region $6(-2681 \mathrm{bp}$ to $-3234 \mathrm{bp})$; whereas a stronger association of $\mathrm{Mi2}$ was seen to the regions 16 and 17 $(-8802 \mathrm{bp}$ to $-9352 \mathrm{bp}$ ) within the $1.5-\mathrm{kb}$ region. We next performed ChIP analysis of lysates from HC11/pcDNA and $\mathrm{HC} 11 / \mathrm{T} 7-\mathrm{MTA} 3$ cells, and found a clear increased recruitment of T7-MTA3 to regions 6 (-2681 bp to -3234

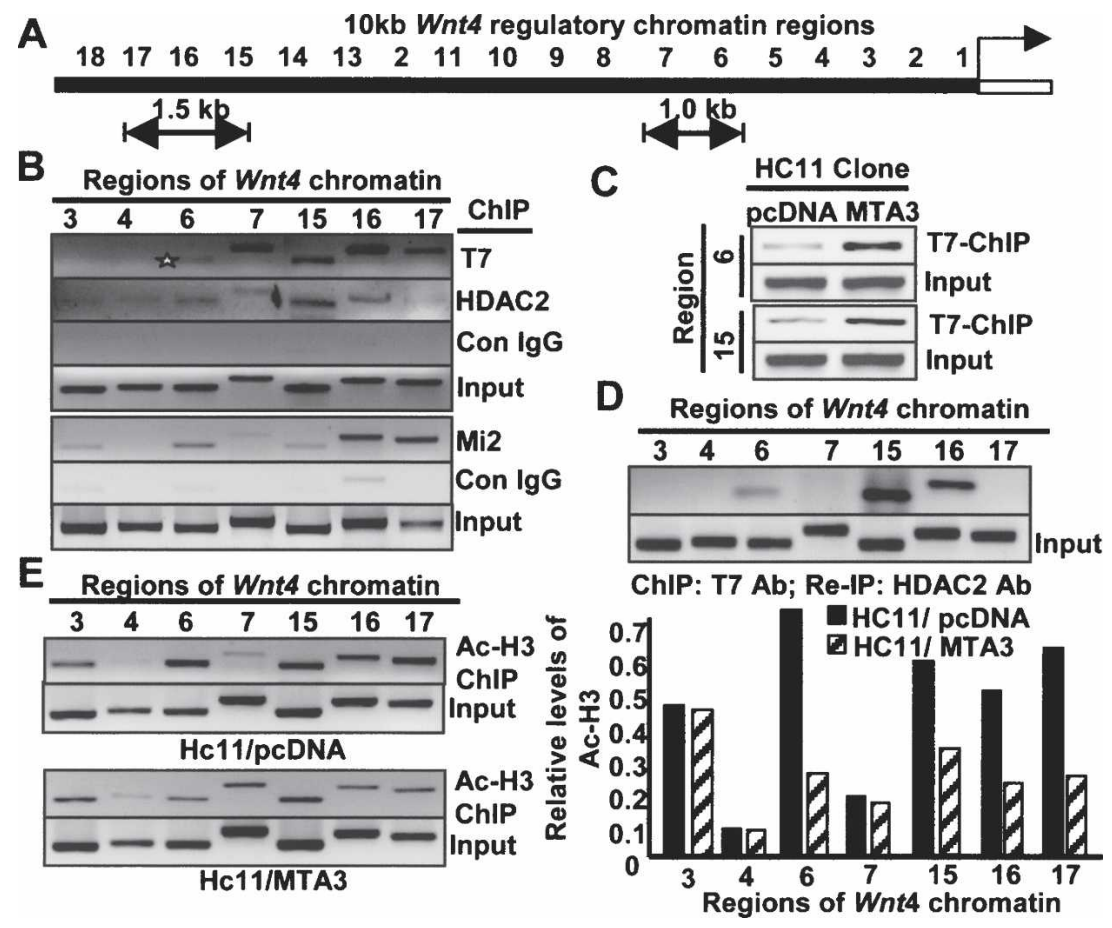

Figure 4. MTA3/NuRD complex targets Wnt4 regulatory chromatin. $(A)$ Schematic diagram of $10-\mathrm{kb}$ regulatory chromatin of mouse Wnt4 gene. (B) ChIP analysis showed T7MTA3, HDAC2, and Mi2 binding to the $1-\mathrm{kb}$ and $1.5-\mathrm{kb}$ regions of the Wnt4 chromatin. Regions 3 and 4 were the negative control. (C) ChIP assay showing differential recruitment of T7-MTA3 to regions 6 and 15 of Wnt4 chromatin in HC11/MTA3 cells (D) A double ChIP assay, first with $\mathrm{T} 7 \mathrm{Ab}$ followed by the HDAC2 Ab for the two regions of Wnt4 chromatin. (E) Status of the acetylated histone H3 in the MTA3-NuRD complex-bound Wnt4 chromatin in the HC11/pcDNA and HC11/MTA3 by ChIP analysis (left) and the relative acetyl-H3 levels at MTA3-binding regions of the Wnt4 chromatin (right). bp) and $15(-7688 \mathrm{bp}$ to $-8243 \mathrm{bp})$ of Wnt4 chromatin in HC11/T7-MTA3 cells over the albeit basal levels in HC11/pcDNA cells (Fig. 4C). HC11/MTA3 clones; the initial ChIP analysis was done anti-T7 mAb to precipitate the T7-MTA3-bound DNA sequences, and the subsequent ChIP analysis was done using the anti-HDAC2 antibody. Results showed taneous association of MTA3 and HDAC2 with the region $6(-2681 \mathrm{bp}$ to $-3234 \mathrm{bp})$ and regions encompassing 15 and $16(-7688 \mathrm{bp}$ to $-8801 \mathrm{bp})$ but not Wnt4 chromatin. Regions 3 and 4 of the Wnt4 chromatin, where no association of MTA3 was seen in ChIP assays were used as negative controls. To specific antibody, and found a considerable decrease in levels of the acetylated $\mathrm{H} 3$ associated with the $1-\mathrm{kb}$ $(-2681 \mathrm{bp}$ to $-3793 \mathrm{bp}$ ) and with the $1.5-\mathrm{kb}$ (-7688 bp to $-9352 \mathrm{bp}$ ) regions of the Wnt4 chromatin in the HC11/ MTA3 cells as compared with the corresponding Wnt4 regions in the HC11/pcDNA cells (Fig. 4E). specific, as there was no recruitment of MTA1 to the above regions of Wnt4 chromatin (Supplementary Fig. S4A), and no association of MTA3 to an unrelated Wnt1 gene chromatin (Supplementary Fig. S4B), further confirming the specificity of MTA3 regulation of Wnt4 chromatin. To assess the functionality of the noted Wnt4 chromatin in the context of MTA3, we cloned the $1-\mathrm{kb}$ and $1.5-\mathrm{kb}$ regions of the Wnt4 into pGL3 basic luciferase vector and tested the ability of MTA3 to affect the Wnt4-luc activity in HC11 by transient and stable overexpression of MTA3, MTA1, or pcDNA. We found that MTA3 but not MTA1 drastically represses Wnt4-luc activity (Supplementary Fig. S5), suggesting a direct role of MTA3 in repressing Wnt4 transcription.

\section{MTA3 suppresses mammary gland ductal side branching during early pregnancy}

Wnt4 is essential for mammary gland development and side branching in early pregnancy (Brisken et al. 2000). We examined the effect of MTA3-TG upon the ductal branching in mammary glands from pregnant day 2 female mice. Similar to the virgin mammary gland, we found reduced branching and BrDU incorporation in the early pregnancy (Supplementary Fig. S6A,B). As Wnt4 was shown to play a major role in mammary gland development downstream from progesterone signaling (Brisken et al. 2000), we tested whether MTA3 could repress the functions of PR. In a luc-based assay conducted in the HC11/ 
pcDNA and HC11/MTA3 cells, MTA3 was found to be potent repressor of progesterone-stimulated activity of PR (Supplementary Fig. S6C). Consistent with a potential suppression of the PR action, we found an inability of progesterone to stimulate Wnt4 expression in the HC11/MTA3 cells as compared with HC11/pCDNA cells (Supplementary Fig. S6D). Next, we tested the ability of progesterone to modulate the level of MTA3, an inhibitor of Wnt4 gene expression (this study). However, we found no effect of progesterone upon the level of MTA3 mRNA in the HC11 cells (Supplementary Fig. $\mathrm{S} 6 \mathrm{E})$. Together, these results suggested that the noticed proliferation defect and hypobranching in the MTA3-TG mammary epithelium might be causally linked with the repressed Wnt4 pathway.

Mammary gland differentiation and morphogenesis is hindered by MTA3 overexpression

Wnt signaling is also required for differentiation processes during organogenesis (Moon et al. 2002). Therefore, we investigated whether the inhibition of Wnt4 pathway in HC11/MTA3 cells would affect their ability to undergo differentiation. One of the physiological effects of the differentiation-stimulating signals in mammary epithelial cells is to accumulate neutral lipid droplets, which can be easily detected by oil red-O staining. We compared the extent of differentiation in the HCl1/ MTA3 clones with the extent in the control HC11/ pcDNA clones in response to the lactogenic hormones dexamethasone, insulin, and prolactin (DIP). We found that $\mathrm{HC} 11 / \mathrm{pcDNA}$ cells underwent complete differentiation, whereas cellular differentiation in HC11/MTA3 clones was considerably suppressed (Fig. 5A).

Whole-mount analysis of the lactating mammary gland from the MTA3-TG mice revealed a profound suppression of mammary gland morphogenesis as compared with the wild-type mice (Fig. 5B). Histologic study of the mammary gland on lactation day 2 by $\mathrm{H} \& \mathrm{E}$ staining showed a lower density of alveolar lobules in the MTA3TG mice than in the wild-type mice, another indicator of a decreased differentiation (Fig. 5B). The inhibitory effect of the MTA3-TG upon differentiation was further validated by a distinct reduction of levels of the phosphorylated Tyr694-STAT5a in the MTA3-TG mice compared with the wild-type mice (Fig. 5B). Since phosphorylated STAT5a has been closely linked to mammary gland differentiation (Liu et al. 1997), our findings were consistent with the idea of an overall suppressed mammary gland differentiation in MTA3-TG mice, possibly by the suppressed expression and secretion of bioactive Wnt4.

Next we examined the status of Wnt4 and its negative regulator MTA3 during mammary gland development in the wild-type mice. Western blot analysis showed an inverse correlation of the levels of Wnt4 and MTA3 protein in the virgin and pregnant mammary glands, and to some extent, in the lactating mammary glands (Fig. 5C). The suppression of the mammary gland morphogenesis in the MTA3-TG mice indicated the possible hindrance of lactation. To evaluate whether MTA3 could affect the expression of milk proteins, we examined its effect on the $\beta$-casein transcription using a $\beta$-casein promoter-luc reporter. A significant repression of the basal as well as DIP-induced $\beta$-casein promoter-luc activity was observed in the HC11/MTA3 cells (Fig. 5D), suggesting a corepressive activity of MTA3. This was supported by

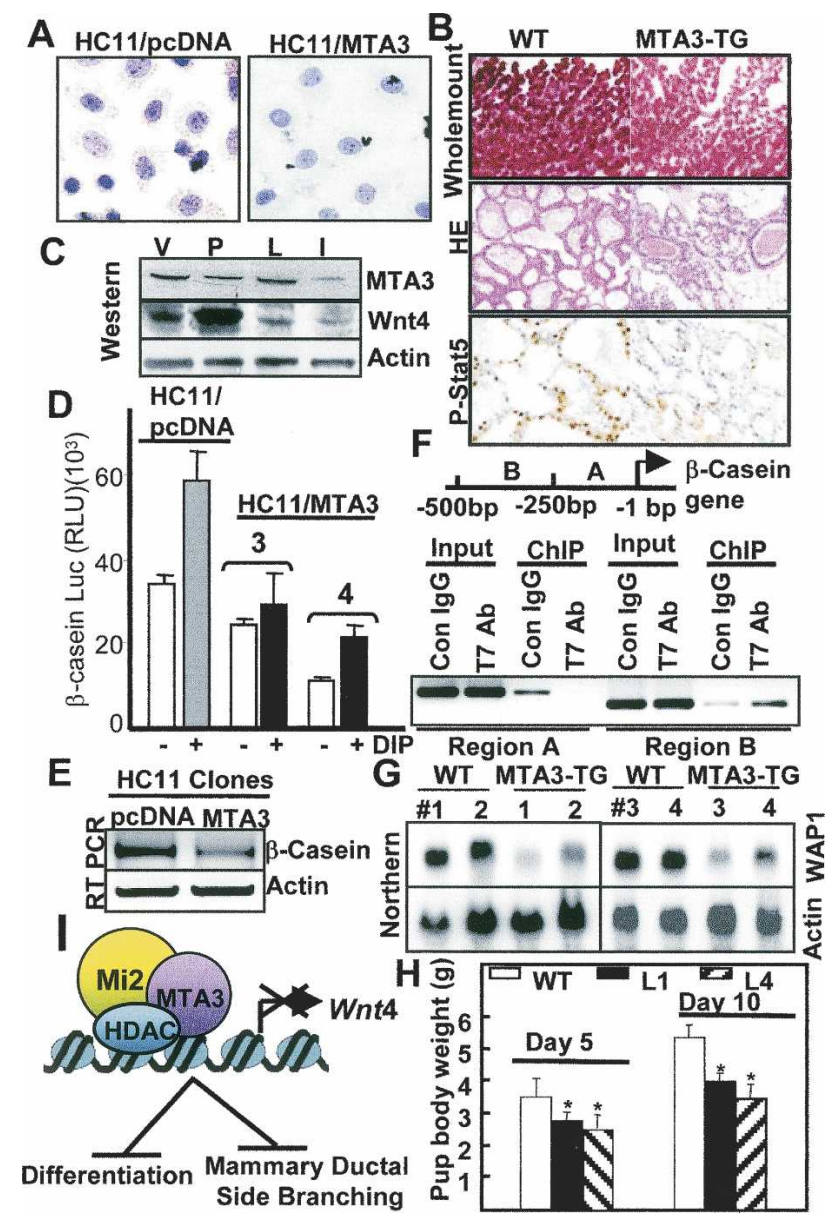

Figure 5. MTA3 suppresses mammary gland differentiation and morphogenesis. (A) Status of the oil red-O-stained neutral lipid droplets in the HC11 clones treated with DIP for $3 \mathrm{~d}$. (B) Whole-mount staining, H\&E staining, and immunostaining for P-STAT5 of mammary tissues at lactation day 2. (C) Status of Wnt4 and MTA3 proteins in various stages of the wild-type mammary gland development. (V) Virgin day (12 wk); (P) pregnancy day 4; (L) lactation day 1 ; (I) involution day 5. (D) Repression of $\beta$-casein-luc activity in $\mathrm{HC} 11$ / MTA3 cells $(E)$ Repression of the levels of $\beta$-casein mRNA in HC11/ MTA3 cells in comparison with the levels in HC11/pcDNA cells. $(F)$ ChIP showing association of MTA3 with a 250-bp region of the $\beta$-casein promoter encompassing -250 bp to -500 bp. (G) Northern blot analysis of the WAP1 in mammary glands from the lactation day 2 wild-type (WT) and MTA3-TG mice. $(H)$ Pup body weight at lactation days 5 and 10 . Values are average weight \pm standard deviation. $P<0.05$ for L1 and L4 according to Student's $t$-test. $(I)$ Schematic diagram of regulation of Wnt4 expression by the MTA3/ NuRD complex and its consequences on mammary gland development.

lower levels of $\beta$-casein mRNA in the HC11/MTA3 clones when compared with HC11/pcDNA cells (Fig. 5E). Next we performed a ChIP analysis, where binding of the MTA3 to $500 \mathrm{bp}$ DNA upstream of the $\beta$-casein gene, a region shown to contain lactogenic-responsive regulatory elements (Saito and Oka 1996), was assessed in the HC11/MTA3 cells. We found a strong association of T7-MTA3 with the region B $(-251$ to $-500 \mathrm{bp})$, while there was no association of the T7-MTA3 promoter region $\mathrm{A}(-1$ to $1250 \mathrm{bp})$ (Fig. $5 \mathrm{~F})$, suggesting a role of MTA3 in the repression of the $\beta$-casein gene.

Since MTA3 transgene resulted in reduced secretion of Wnt 4 , we explored the effect of Wnt 4 expression on $\beta$-ca- 
sein gene in the HC11 cells. Transient overexpression of Wnt4 resulted in no noticeable change in the $\beta$-casein luc activity (Supplementary Fig. S7A), whereas Wnt4 knockdown with Wnt4-specific siRNA showed a modest reduction in the $\beta$-casein luc activity upon DIP treatment (Supplementary Fig. S7B). To further decipher the effect of Wnt4 on the differentiation of mammary cells, Wnt4 expression was silenced in the HC11 cells using Wnt4 siRNA and cells were stimulated with DIP for 48 $\mathrm{h}$. Oil red-O staining showed a noticeable decrease in the accumulation of neutral oil droplets in cells with Wnt4 knockdown in comparison with the control cells (Supplementary Fig. S7C). The effect was not as pronounced as in the HC11/MTA3 clones (Fig. 5A), where it might be due to long-term depletion of Wnt4.

In addition to the $\beta$-casein gene, we also observed reduced steady-state mRNA levels of another marker of lactogenesis, whey acidic protein 1 (WAP1) in mammary glands of four independent MTA3-TG mice in comparison with the levels of WAP1 mRNA in the wild-type mice (Fig. 5G). The repressive effect of MTA3 overexpression on the $\beta$-casein and WAP1 suggested a potential lactation defect in the MTA3-TG mice. This was supported by the presence of milk in the belly of 6-d-old pup born to the wild-type female mice, whereas there was no or albeit milk in the belly of age-matched pups from the MTA3-TG female (Supplementary Fig. S8). The body weight of the pups in two MTA3-TG founder lines, 5 and $10 \mathrm{~d}$ after birth, was consistently lower than was the weight of wild-type pups of the same age (Fig. $5 \mathrm{H}$ ), presumably because of the reduced milk production in the MTA3-TG mice. These findings supported the notion that a reduction in the level of Wnt 4 may be closely associated with a reduced differentiation, which along with reduction in milk proteins may contribute to the observed lactation defect in the MTA3-TG mice.

A principle finding of this study is an inhibitory effect of MTA3 transgene on the ductal side branching in mammary glands due to the suppression of Wnt4 signaling resulting in reduced cell proliferation and differentiation. MTA3 was a direct repressor of Wnt4 transcription, and this repression could not be rescued by progesterone signaling in HC11 mammary epithelial cells. It is of interest to note that the effects of MTA3 overexpression in the mammary gland development were opposite of those seen in the MTA1-TG mice. Overexpression of MTA1-TG resulted in an excessive branching and precocious development of the mammary gland due to a higher degree of proliferation and differentiation (Bhageri-Yarmand et al. 2004). These findings also support the notion of physiologic differences between the MTA family members, and lack of functional redundancy between MTA3 and MTA1. In summary, we identified Wnt4 as a novel target of MTA3, making it the first direct corepressor of Wnt4 transcription in a physiologic setting.

\section{Materials ands methods}

Generation of transgenic mice and genotyping

An MMTV-human MTA3-TG construct was created by subcloning T7. tagged MTA3 cDNA into MMTV-SV40-BssK vector (Bagheri-Yarmand et al. 2004), and summarized in the Supplemental Material.

Whole-mount analysis and histology

The whole-mount analysis, and histology was performed as previously described (Bagheri-Yarmand et al. 2004), and summarized in the Supplemental Material.
In situ hybridization, $R T-P C R$, and ChIP

Digoxigenin-labeled in vitro transcripts were generated from plasmid containing mouse Wnt4 fragments (Brisken et al. 2000). RT-PCR and ChIP were performed as summarized in the Supplemental Material.

\section{Acknowledgments}

We thank Andrew McMahon for providing the mouse Wnt4 fragment (via Richard Behringer) for in situ studies, and Joseph Mascarenhas for promoter cloning. This study was supported in part by The Norman Brinkler Award for Research Excellence, and NIH Grant CA98823 (to R.K).

\section{References}

Bagheri-Yarmand, R., Talukder, A.H., Wang, R.A., Vadlamudi, R.K., and Kumar, R. 2004. Metastasis-associated protein 1 deregulation causes inappropriate mammary gland development and tumorigenesis. Development 131: 3469-3479.

Bradbury, J.M., Edwards, P.A., Niemeyer, C.C., and Dale, T.C. 1995 wnt-4 expression induces a pregnancy-like growth pattern in reconstituted mammary glands in virgin mice. Dev. Biol. 170: 553-563.

Brisken, C., Heineman, A., Chavarria, T., Elenbaas, B., Tan, J., Dey, S.K., McMahon, J.A., McMahon, A.P., and Weinberg, R.A. 2000. Essential function of wnt-4 in mammary gland development downstream of progesterone signaling. Genes \& Dev. 14: 650-654.

Civenni, G., Holbro, T., and Hynes, N.E. 2003. Wnt1 and Wnt5a induce cyclin D1 expression through ErbB1 transactivation in HC11 mammary epithelial cells. EMBO Rep. 4: 166-171.

Devagan, V., Mammucari, C., Millar, S.E., Brisken, C., and Dotto, G.P. 2005. p21WAF1/Cip1 is a negative transcriptional regulator of wnt4 expression downstream of Notch1 activation. Genes \& Dev. 9: 14851495.

Fujita, N., Jaye, D.L., Kajita, M., Geigerman, C., Moreno, C.S., and Wade, P.A. 2003. MTA3, a Mi-2/NuRD complex subunit, regulates an invasive growth pathway in breast cancer. Cell 113: 207-219.

Fujita, N., Jaye, D.L., Geigerman, C., Akyildiz, A., Mooney, M.R., Boss, J.M., and Wade, P.A. 2004. MTA3 and the Mi-2/NuRD complex regulate cell fate during B lymphocyte differentiation. Cell 119: 75-86.

Humphreys, R.C. and Rosen, J.M. 1997. Stably transfected HC11 cells provide an in vitro and in vivo model system for studying Wnt gene function. Cell Growth Differ. 8: 839-849.

Kumar, R. 2003. Another tie that binds the MTA family to breast cancer. Cell 113: 142-143.

Kumar, R., Wang, R.A., and Bagheri-Yarmand, R. 2003. Emerging roles of MTA family members in human cancers. Semin. Oncol. 30: 30-37.

Liu, X., Robinson, G.W., Wagner, K.U., Garrett, L., Wynshaw-Boris, A., and Hennighausen, L. 1997. Stat5a is mandatory for adult mammary gland development and lactogenesis. Genes \& Dev. 11: 176-186.

Lydon, J.P., DeMayo, F.J., Funk, C.R., Mani, S.K., Hughes, A.R., Montgomery Jr., C.A., Shyamala, G., Conneely, O.M., and O'Malley, B.W. 1995. Mice lacking progesterone receptor exhibit pleiotropic reproductive abnormalities. Genes \& Dev. 9: 2266-2278.

Mishra, S.K., Talukder, A.H., Gururaj, A.E., Yang, Z., Singh, R.R., Mahoney, M.G., Franci, C., Vadlamudi, R.K., and Kumar, R. 2004. Upstream determinants of estrogen receptor- $\alpha$ regulation of metastatic tumor antigen 3 pathway. J. Biol. Chem. 279: 32709-32715.

Moon, R.T., Bowerman, B., Boutros, M., and Perrimon, N. 2002. The promise and perils of wnt signaling through $\beta$-catenin. Science 296: 1644-1646.

Nusse, R. and Varmus, H.E. 1992. Wnt genes. Cell 69: 1073-1087.

Pencil, S.D., Toh, Y., and Nicolson, G.L. 1993. Candidate metastasisassociated genes of the rat $13762 \mathrm{NF}$ mammary adenocarcinoma. Breast Cancer Res. Treat. 25: 165-174.

Robinson, G.W., Hennighausen, L., and Johnson, P.F. 2000. Side-branching in the mammary gland: The progesterone-wnt connection. Genes \& Dev. 14: 889-894.

Saito, H. and Oka, T. 1996. Hormonally regulated double- and singlestranded DNA-binding complexes involved in mouse $\beta$-casein gene transcription. J. Biol. Chem. 271: 8911-8918. 


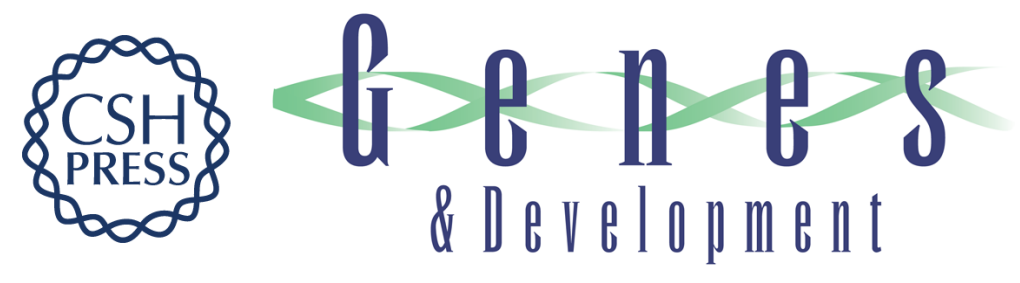

\section{Metastatic tumor antigen 3 is a direct corepressor of the Wnt4 pathway}

Hao Zhang, Rajesh R. Singh, Amjad H. Talukder, et al.

Genes Dev. 2006, 20: originally published online October 18, 2006

Access the most recent version at doi:10.1101/gad.1461706

\section{Supplemental http://genesdev.cshlp.org/content/suppl/2006/10/17/gad.1461706.DC1 Material}

References This article cites 18 articles, 9 of which can be accessed free at: http://genesdev.cshlp.org/content/20/21/2943.full.html\#ref-list-1

\section{License}

Email Alerting

Receive free email alerts when new articles cite this article - sign up in the box at the top Service 